\title{
2017 Acknowledgments
}

Psychology of Popular Media Culture is very fortunate to have a wide pool of talented reviewers to complement the editorial board. The Editor would like to thank the following scholars for contributing their expertise and energy.

Aimee Adam

Baxter Lindsey Morrison Adams

Sandy Alexandraki

Phyllis Anastasio

Shayne Aquino

Hanan Asghar

Eleni-Marina Ashikali

Jennifer Stevens Aubrey

Katherine Aumer

Elizabeth Baker

Jaime Banks

Joel Billieux

Max Birk

Kim Bissell

Erik Black

Nevfel Boz

Johannes Breuer

Britney G. Brinkman

Jeanne Brockmyer

Steven Brown

Ngoc Hong Bui

Keith Campbell

Reuben Mikhael

Castagno

Joshua Childs

Kyung-Shick Choi

Sally Chung

Vincent Cicchirillo

Annabel Cohen

M. Nicole Coleman

Mark Coulson

Ryan Scott Creech

David Cropley

Di Cui

Gerald Cupchik

Elizabeth A. Daniels

Shayn Davidson

Leslie Alison Davis

Jaye L. Derrick

Benjamin B. DeVore
Anne Dickmeis

Don Diefenbach

Edward Downs

Michelle Drouin

Aaron Drummond

Robert Andrew Dunn

Jennifer Dyer-Seymour

Carla Earhart

Morgan E. Ellithorpe

Malte Elson

Elisabeth Engelberg

Sarah Erickson

Adam Feltz

Arienne Ferchaud

Antonis Gardikiotis

Judith Gibbons

Bryan Gibson

Kuba Glazek

Sara Gluck

Thalia R. Goldstein

Gordon Goodman

John Goodwin

Melinda A. Green

Dara Greenwood

Tobias Greitemeyer

Christopher Groves

Alice Hall

Tammy Harpel

Veronica Hefner

Sylvia Herbozo

Joseph Hilgard

August John Hoffman

Jessica D. Hoffmann

Cynthia Hoffner

Jim Houran

Tom Hummer

Nicole E. Iannone

Joanne Ingram
Ruud Jacobs

Sophie Helga Janicke

Michael Jenkins-Guarnieri

Sven Jöckel

Benjamin K. Johnson

Jessie Quintero Johnson

Satoshi Kanazawa

Hansika Kapoor

Sarah A. Kass

Jen Katz-Buonincontro

Linda K. Kaye

David Kidd

Janna Kim

J. Matias Kivikangas

Monica Koehn

Hanna Krasnova

Linda Kreger

Valerie Ellen Kretz

Nicolle Lamerichs

Travis Langley

Samuel Leistedt

Eric Li

Julia R. Lippman

Kathryn L. Lookadoo

Sara Magee

Julia A. Maier

Raymond Mar

Brandon T. McDaniel

Andrea McDonnell

D. Cricket Meehan

André Melzer

Altaf Merchant

Brandon Miller

Dirk Oliver Mügge

Karla Murdock

Jessica Gall Myrick

Weihua Niu

Oluyinka Ojedokun 
Mary Beth Oliver Lucia O'Sullivan

\begin{abstract}
Alanna Peebles
Robert Perl

Halley M. Pontes
\end{abstract}

Arthur Raney

Apara Ranjan

Rabindra Ratan

Leonard Reinecke

Grant Rich

Karyn Riddle

Christine Elizabeth

Rittenour
Melanie Domenech

Rodriguez

Nicolas Ruth

Goal Auzeen Saedi

Meghan Sanders

Angeline Sangalang

E. Glenn Schellenberg

Michael Schmierbach

Christina Schumann

Pavica Sheldon

Jennie K. Singer

Jeffrey Smith

Stephanie Spielmann

Gayle Stever

Chris Stiff
Royette Tavernier

Morgan Tear

Michael Toohey

Raphael Travis

Jessica Troilo

Mina Tsay-Vogel

Jean Twenge

Jan Van den Bulck

Indre Viskontas

Erin Alyssa Vogel

Jeffrey Wimmer

Jason Young 